\title{
ADJUSTMENT OF TOPOLOGICAL CONCORDANCES AND EXTENSIONS OF HOMEOMORPHISMS OVER PINCHED COLLARS
}

\author{
T. B. RUSHING ${ }^{1}$
}

\begin{abstract}
Under special conditions, it is shown how to adjust topological concordances so as to leave certain sets fixed. Also, a lemma is given which establishes the existence of pinched collars and bicollars under specified circumstances. These two results are then combined to show how to obtain nice extensions of certain homeomorphisms.
\end{abstract}

If $X$ and $Y$ are topological spaces and $h_{0}$ and $h_{1}$ are embeddings of $X$ into $Y$, a topological concordance between $h_{0}$ and $h_{1}$ is an embedding $H: X \times I \rightarrow Y \times I \quad(I=[0,1])$ such that $H(x, 0)=\left(h_{0}(x), 0\right)$ and $H(x, 1)=\left(h_{1}(x), 1\right)$ for all $x \in X$. If $A \subset X$, a topological concordance $H$ between $h_{0}: X \rightarrow Y$ and $h_{1}: X \rightarrow Y$ is fixed on $A$ if $H(x, t)=\left(h_{0}(x), t\right)$ for all $(x, t) \in A \times I$.

The following theorem generalizes the principal result of [4], i.e., Theorem 4.1 of [4]. In fact, the following theorem reduces to Theorem 4.1 of [4], when $M$ is a point.

Theorem. Let $M^{m}$ be an m-dimensional PL-manifold and let $Q^{q}$, $q-m \geqq 3$, be a q-dimensional PL-manifold. Suppose that $M$ is $(2 m-q+2)$-connected, $\partial M$ is $(2 m-q+1)$-connected (or empty), and $Q$ is $(m+1)$-connected. Let $f: M \rightarrow \operatorname{Int} Q$ be a locally flat embedding. If $h_{0}$ and $h_{1}$ are topological homeomorphisms of $Q$ onto itself which leave $f(M)$ fixed and which are topologically concordant, then there is a topological concordance between $h_{0}$ and $h_{1}$ which is fixed on $f(M)$.

REMARK 1. The above theorem can be generalized in a straightforward way to permit $f$ to be certain allowable embeddings. We leave the calculation of the necessary connectivity conditions to the reader.

REMARK 2. A generalization of the piecewise linear version of the theorem has been obtained in a joint paper with L. S. Husch [6] since the submission of this paper. Also, the piecewise linear version

Received by the editors March 28, 1969.

AMS subject classifications. Primary 5702, 5705, 5478, 5560, 5570.

Key words and phrases. Concordance, extension, pinched collar, PL manifold, locally flat.

1 Partially supported by the National Science Foundation. 
of the theorem where the concordances of the hypothesis and conclusion are replaced by isotopies is proved in [6]. A good result would be the topological version of the isotopy theorem. Such a proof would follow by the techniques of [6] if a "level-preserving taming theorem" were available.

Before proving the theorem, we will consider two corollaries. Corollary 1 follows easily from the theorem and the fact that every orientation preserving homeomorphism of $S^{n}, n \geqq 5$, is isotopic to the identity. (The preceding fact follows from the recent work of R. C. Kirby, L. C. Siebenmann and C. T. C. Wall.)

Corollary 1. Suppose that $X^{k}$ is a locally flat $k$-cell or $k$-sphere in $S^{n}$ (or $\left.E^{n}\right), n-k \geqq 3, n \geqq 5$, and that $h$ is an orientation preserving homeomorphism of $S^{n}$ (or $\left.E^{n}\right)$ which restricts to the inclusion on $X$. Then, $h$ is topologically concordant to the identity keeping $X$ fixed.

Suppose that $M$ is a submanifold of the manifold $N$. Then, $M$ is said to be collared in $N$ if there is an embedding $h$ carrying $M \times I$ onto a neighborhood of $M$ such that $h(x, 0)=x$ for all $x \in M$. If $M$ can be covered by a collection of open subsets (relative to $M$ ) each of which is collared in $N$, then $M$ is locally collared in $N$. Suppose that $X$ is a closed subset of $M$. If there is a homeomorphism

$$
h:(M \times[-1,1]) /[(x, t) \approx(x, 0) \text { if } x \in X,-1 \leqq t \leqq 1] \rightarrow N
$$

such that $h([(x, 0)])=x$ and

$P(X, M, N)$

$$
=h((M \times[-1,1]) /[(x, t) \approx(x, 0) \text { if } x \in X,-1 \leqq t \leqq 1])
$$

is a neighborhood of $M-X$ in $N$, then we call $P(X, M, N)$ a bicollar of $M$ in $N$ pinched at $X$. Pinched collars are defined similarly.

Pinched (BI)collar Lemma. (a) Let $X$ be a closed subset of $a$ manifold $M$ which is contained in a manifold $N$. If $M-X$ is locally collared in $N$, then there is a collar of $M$ in $N$ pinched at $X$.

(b) Let $X$ be a closed subset of the boundary of a manifold $M$. Then, there is a collar of $\mathrm{Bd} M$ in $M$ pinched at $X$.

(c) Let $M$ be an (n-1)-submanifold of an n-manifold $N$ and let $X$ be a closed subset of $M$. If $M-X$ is a two-sided, connected, locally flat submanifold of the interior of $N$, then there is a bicollar of $M$ in $N$ pinched at $X$.

The above lemma can be established by an argument which makes use of results of [1]. 
EXAMPLE. (It is instructive to consider generalizations of the following example and their relationship to extension theorems such as Corollary 2 below.) Let $M$ be the central circle of a Möbius band $N$ and let $X$ be a point of $M$. Then, $M$ is locally flat in $N$, but $M$ is not bicollared in $N$. However, there is a bicollar of Min $N$ pinched at $X$ by the Pinched Bicollar Lemma.

The following corollary is quite useful and is typical of extension theorems which can be obtained from the theorem and the Pinched Bicollar Lemma.

Corollary 2. Suppose that $\Sigma^{n}, n \geqq 5$, is a two-sided locally flat $n$-sphere in the interior of the $(n+1)$-manifold $V^{n+1}$. Let $X^{k} \subset \Sigma$ be a $k$-cell or $k$-sphere, $n-k \geqq 3$, which is locally flat in $\Sigma$ and let $h$ be an orientation preserving homeomorphism of $\Sigma$ which is the identity on $X$. Then, $h$ extends to a homeomorphism of $V$ which is the identity outside of a bicollar of $\Sigma$ pinched at $X$.

REMARK 3. Instead of applying the Pinched Bicollar Lemma for Corollary 2, one can obtain the necessary pinched bicollar by applying some machinery in the case $V$ is a PL manifold. First, use Theorem 3 of [2], announced in [7], to get a homeomorphism $h: V \rightarrow \rightarrow V$ such that $h(\Sigma)$ and $h(X)$ are subpolyhedra of $V$. Next, the corollary of [3] gives us a homeomorphism $g: V \rightarrow \rightarrow V$ such that $g h(\Sigma)$ is a PL-bicollared subpolyhedron of $V$.

REMARK 4. For an application of Corollary 2 see the last paragraph of the proof of Theorem 1 of [10]. Now let $N$ be a regular neighborhood of $g h(\Sigma) \bmod g h(X)$ in $V$ in the sense of Marshal Cohen. (See Definition 3.5 of [8].) Then, it follows from Lemma 3.9 of [8] that $N$ is a bicollar of $g h(\Sigma)$ pinched at $g h(X)$. Hence, $g h^{-1}(N)$ is the desired pinched bicollar.

Problem. Obtain generalizations of Corollary 2 (other than the obvious ones which follow from this paper).

Proof of THE Theorem. Let $H: Q \times I \rightarrow Q \times I$ be a topological concordance between $h_{0}$ and $h_{1}$. Define $F: M \times I \rightarrow Q \times I$ by $F(x, t)$ $=(f(x), t), x \in M, t \in I$. We will be finished if we can find a homeomorphism $h: Q \times I \rightarrow \rightarrow Q \times I$ such that $h \mid Q \times\{0,1\}=1$ and $h H f=F$, because then $h H$ will be a topological concordance between $h_{0}$ and $h_{1}$ which is fixed on $f(M)$.

By Theorem 1 of [9], we can get a homeomorphism $g: Q \rightarrow \rightarrow Q$ such that $g f: M \rightarrow Q$ is PL. Now, define $G: Q \times I \rightarrow \rightarrow Q \times I$ by $G(x, t)$ $=(g(x), t)$. Then $G F: M \times I \rightarrow Q \times I$ is PL. Also, $G H F: M \times I \rightarrow Q \times I$ is a locally flat embedding such that $G H F|M \times\{0,1\}=G F| M \times\{0,1\}$. By Theorem 1 and Addendum 1.1 of [9], there is a homeomorphism 
$K: Q \times I \rightarrow \rightarrow Q \times I$ such that $K \mid \partial(Q \times I)=1$ and $K G H F: M \times I \rightarrow Q \times I$ is PL. Now, the PL embeddings $G F \mid \partial M \times I: \partial M \times I \rightarrow Q \times I$ and $K G H F \mid \partial M \times I: \partial M \times I \rightarrow Q \times I$ agree on $\partial M \times\{0,1\}=\partial(\partial M \times I)$ $=(G F \mid \partial M \times I)^{-1} \partial(Q \times I)=(K G H F \mid \partial M \times I)^{-1} \partial(Q \times I)$. Thus, these two embeddings are allowable and they are homotopic keeping $\partial(\partial M \times I)$ fixed since $\partial M \times I$ is m-dimensional and $Q$ is $(m+1)$ connected. Also, $\partial M \times I$ is $(2 m-q+1)$-connected since $\partial M$ is $(2 m-q+1)$-connected and $Q \times I$ is $(2 m-q+3)$-connected since $q-m \geqq 3 \Rightarrow q-2>m \Rightarrow m+q-2>0 \Rightarrow(m+1)-(2 m-q+3)>0 \Rightarrow m+1$ $>2 m-q+3$. Thus, we can apply Theorem 4 of [5] to obtain a PL homeomorphism $J: Q \times I \rightarrow Q \times I$ such that $J \mid \partial(Q \times I)=1$ and $J K G H F|\partial M \times I=G F| \partial M \times I$. It is easy to check that we can again apply Theorem 4 of [5] to $J K G H F: M \times I \rightarrow Q \times I$ and $G F: M \times I$ $\rightarrow Q \times I$ to get a PL-homeomorphism $L: Q \times I \rightarrow \rightarrow Q \times I$ such that $L \mid \partial(Q \times I)=1$ and $L J K G H F=G F$. Hence, $h=G^{-1} L J K G$ is the homeomorphism that we sought and the proof is complete.

\section{REFERENCES}

1. M. Brown, Locally flat embeddings of topological manifolds, Ann. of Math. (2) 75 (1962), 331-341. MR 24 \#A3637.

2. J. C. Cantrell and T. B. Rushing, On low codimensional taming, Proc. Georgia Topology Conference, 1969 (to appear).

3. M. Cohen and D. Sullivan, On the regular neighborhood of a two-sided submanifold, (to appear).

4. H. Gluck, Restriction of isotopies, Bull. Amer. Math. Soc. 69 (1963), 78-82. MR $26 \# 750$.

5. J. F. P. Hudson, Concordance and isotopy of PL embeddings, Bull. Amer. Math. Soc. 72 (1966), 534-535. MR 33 \#1857.

6. L. S. Husch and T. B. Rushing, Restrictions of isotopies and concordances, Michigan Math. J. 16 (1969), 303-307.

7. T. B. Rushing, Taming codimension three embeddings, Bull. Amer. Math. Soc. 75 (1969), 815-820.

8. - Taming embeddings of certain polyhedra in codimension three, Trans. Amer. Math. Soc. 145 (1969), 87-103.

9. - Locally flat embeddings of $\mathrm{PL}$ manifolds are $\mathrm{\epsilon}$-tame in codimension three, Proc. Georgia Topology Conference, 1969 (to appear).

10. - Unknotting unions of cells, Pacific J. Math 32 (1970), 521-525.

University of Georgia, Athens, Georgia 30601

University of Utah, Saly Lake City, Utah 84112 